\title{
V ARIATIONAL COMPARISON THEOREM AND PERTURBATIONS OF NONLINEAR SYSTEMS
}

\author{
G. S. LADDE
}

ABSTRACT. By employing vector Lyapunov-like functions and the theory of systems of differential inequalities, a variational comparison theorem and generalized variation of constants formula are developed. Furthermore, the usefulness of the variational comparison theorem is exhibited by giving applications to stability problems.

1. Introduction. Two of the most useful methods available for studying the qualitative and quantitive behavior of the solutions of a nonlinear system of differential equations are the second method of Lyapunov, and the comparison with a suitable unperturbed system, namely, linear or nonlinear and the use of the variation of constants formula. These methods have been used successfully to study a variety of problems in a unified way. The excellent source for these methods and their applicability can be found in a recent monograph of Lakshmikantham and Leela [3].

An accurate study of perturbations of differential systems depends upon not only the nature of perturbations but also the method of study. The techniques involved in both of the above indicated methods dictate that we measure the perturbations in terms of its norm and thereby destroy the ideal nature of perturbations.

Recently, an attempt was made by Bernfeld, Ladde and Lakshmikantham [1] to obtain perturbation results in which perturbations were not measured in norm. However, the" method involves the construction of smooth Lyapunov functions on the basis of a certain given behavior of an unperturbed system and its use as a Lyapunov function for a perturbed system.

The purpose of this paper is to develop a suitable technique by means of which we can preserve the ideal nature of perturbations and study the stability properties of perturbed systems. In $\$ 2$, by employing vector Lyapunov-like functions and the theory of systems of differential inequalities, a variational comparison theorem and generalized variation of constants formula are developed. Note that the variational comparison theorem relates the solutions of perturbed, unperturbed and auxiliary equations. This import-

Received by the editors April 3, 1974 and, in revised form, August 6, 1974. $34 \mathrm{D} 10$.

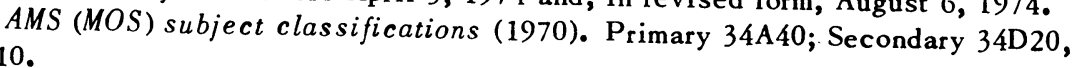

Key words and phrases. Differential inequality, generalized variation of constants formula, maximal solution, stability, variational comparison theorem, $V$-stability, vector Lyapunov-like function. 
ant fact is advantageous for discussing qualitative behavior of the perturbed system. In $\$ 3$, we introduce the notion of $V$-stability and give, as applications, a result on the stability of motion. Examples are worked out to demonstrate the fruitfulness of our results.

2. Basic results. Consider the system of differential equations

$$
x^{\prime}=f(t, x), \quad x\left(t_{0}\right)=x_{0},
$$

and its corresponding perturbed system

$$
y^{\prime}=f(t, y)+R(t, y), \quad y\left(t_{0}\right)=y_{0} .
$$

Assume that $f$ and $R$ in (2.1) and (2.2) satisfy the following hypotheses:

$\left(\mathrm{H}_{1}\right) f \in C\left[R^{+} \times R^{n}, R^{n}\right]$ and $f_{x}$ exists and is continuous on $R^{+} \times R^{n}$, where $R^{+}=[0, \infty)$ and $R^{n}$ is $n$-dimensional Euclidean space with a convenient norm $\|\cdot\|$.

$\left(\mathrm{H}_{2}\right) R \in C\left[R^{+} \times R^{n}, R^{n}\right]$.

It is well known that under $\left(\mathrm{H}_{1}\right)$, for any $\left(t_{0}, x_{0}\right) \in R^{+} \times R^{n}$, there exists a unique solution $x\left(t, t_{0}, x_{0}\right)$ of (2.1) and furthermore, $x\left(t, t_{0}, x_{0}\right)$ is continuously differentiable with respect to $\left(t_{0}, x_{0}\right)$. Let $\phi\left(t, t_{0}, x_{0}\right)$ denote the fundamental solution of the variational system associated with (2.1). For more details see [3].

We shall also be concerned with the function $V \in C\left[R^{+} \times R^{n}, R^{m}\right]$ and the auxiliary differential system

$$
u^{\prime}=g(t, u), \quad u\left(t_{0}\right)=u_{0} .
$$

We shall assume that equation (2.3) and the function $V$ satisfy the following hypotheses:

$\left(\mathrm{H}_{3}\right) V \in C\left[R^{+} \times R^{n}, R^{m}\right]$ and $V_{x}$ exists and is continuous for $(t, x) \epsilon$ $R^{+} \times R^{n}$

$\left(\mathrm{H}_{4}\right) g \in C\left[R^{+} \times R^{m}, R^{m}\right], g(t, u)$ is quasimonotone nondecreasing in $u$ for each $t \in R^{+}$.

$\left(\mathrm{H}_{5}\right)$ Let $r\left(t_{,} t_{0}, u_{0}\right)$ be the maximal solution of the auxiliary equation (2.3) existing for $t \geq t_{0}, t_{0} \in R^{+}$.

$$
\left(\mathrm{H}_{6}\right) \text { For }(s, y) \in R^{+} \times R^{n}, t \geq s \geq t_{0}, t_{0} \in R^{+} \text {, }
$$

$$
V_{x}(t, x(t, s, y)) \phi(t, s, y) R(s, y) \leq g(s, V(t, x(t, s, y))),
$$

where $x(t, s, y)$ is the solution of (2.1) through $(s, y)$.

The following lemma is a natural extension of variation of constants formula for nonlinear system of differential equations.

Lemma 2.1 (Generalized variation of constants formula). Let hypoth- 
eses $\left(\mathrm{H}_{1}\right),\left(\mathrm{H}_{2}\right)$ and $\left(\mathrm{H}_{3}\right)$ be satisfied and let $x(t)=x\left(t, t_{0}, y_{0}\right)$ be the solution of (2.1) existing for $t \geq t_{0}$, and $y(t)=y\left(t, t_{0}, y_{0}\right)$ be any solution of (2.2). Then,

(2.5) $V(t, y(t))=V(t, x(t))+\int_{t_{0}}^{t} V_{x}(t, x(t, s, y(s))) \phi(t, s, y(s)) R(s, y(s)) d s$

Proof. Let $x(t, s, y(s))$ be the solution of (2.1) through $(s, y(s))$ and $y(s)=y\left(s, t_{0}, y_{0}\right)$ be any solution of (2.2) through $\left(t_{0}, y_{0}\right)$. From hypotheses $\left(\mathrm{H}_{1}\right),\left(\mathrm{H}_{2}\right),\left(\mathrm{H}_{3}\right)$ and Theorem 2.5.3 in [3], we have

$$
\begin{aligned}
\frac{d V}{d s}(t, x(t, s, y(s))) & =V_{x}(t, x(t, s, y(s))) \frac{d x}{d s}(t, s, y(s)) \\
& =V_{x}(t, x(t, s, y(s))) \phi(t, s, y(s)) R(s, y(s)) .
\end{aligned}
$$

Noting that $x\left(t, t, y\left(t, t_{0}, y_{0}\right)\right)=y\left(t, t_{0}, y_{0}\right)$, by integrating (2.6) from $t_{0}$ to $t$, we obtain (2.5). The proof is complete.

Remark 2.1. Note that, if $V(t, x)=x$, then the previous lemma reduces to Theorem 2.6.3 in [3] as a very special case.

Reinark 2.2. If $V(t, x)=\|x\|^{2}$, then relation (2.5) becomes

$$
\|y(t)\|^{2}=\|x(t)\|^{2}+2 \int_{t_{0}}^{t} x(t, s, y(s)) \cdot \phi(t, s, y(s)) R(s, y(s)) d s .
$$

Lemma 2.2. Assume that all the hypotheses of Lemma 2.1 hold. Then

$$
\begin{aligned}
V(t, y(t) & -x(t)) \\
& =V(t, 0)+\int_{t_{0}}^{t} V(t, x(t, s, y(s))-x(t)) \phi(t, s, y(s)) R(s, y(s)) d s .
\end{aligned}
$$

Proof. By following the proof of Lemma 2.1, we have the relation $\frac{d V}{d s}(t, x(t, s, y(s))-x(t))=V_{x}(t, x(t, s, y(s))-x(t)) \phi(t, s, y(s)) R(s, y(s))$. After integration, this relation yields (2.8).

Remark 2.3. Similarly, a lemma analogous to Theorem 2.6.4 in [3] can be formulated very easily.

We shall state and prove a comparison theorem which will be more advantageous, in some situations, to study the qualitative behavior of (2.2) relative to (2.1).

Theorem 2.1 (Variational comparison theorem). Assume that hypotheses $\left(\mathrm{H}_{1}\right)-\left(\mathrm{H}_{6}\right)$ hold. If $x(t)=x\left(t, t_{0}, y_{0}\right)$ is a solution of (2.1) such that

$$
V(t, x(t)) \leq u_{0},
$$

then for any solution $y(t)=y\left(t, t_{0}, y_{0}\right)$ of (2.2), we have

$$
V(t, y(t)) \leq r\left(t, t_{0}, u_{0}\right), \quad t \geq t_{0} .
$$


Proof. By following the proof of Lemma 2.1, we have

$$
\frac{d V}{d s}(t, x(t, s, y(s)))=V_{x}(t, x(t, s, y(s))) \phi(t, s, y(s)) R(s, y(s)) .
$$

From (2.4) and (2.11), we get

$$
\frac{d V}{d s}(t, x(t, s, y(s))) \leq g(s, V(t, x(t, s, y(s)))) .
$$

Set

$$
m(s)=V(t, x(t, s, y(s))), \quad m\left(t_{0}\right)=V(t, x(t)),
$$

then, 'in view of (2.11) and (2.12), we have

$$
m^{\prime}(s) \leq g(s, m(s)) .
$$

From (2.9), (2.13), (2.14), and Corollary 1.7.1 in [3], we obtain

$$
m(s) \leq r\left(s, t_{0}, u_{0}\right) \quad \text { for } t \geq s \geq t_{0}
$$

which yields (2.10), in view of the definition of $m(s)$ and the fact that $x\left(t, t, y\left(t, t_{0}, y_{0}\right)\right)=y\left(t, t_{0}, y_{0}\right)$. This completes the proof of the theorem.

Remark 2.4. Note that the comparison Theorem 2.1 is not exactly like the earlier comparison theorems [3]. This theorem relates the solutions of the three differential equations, namely, unperturbed system (2.1), perturbed system (2.2) and auxiliary system (2.3). On the other hand the usual comparison theorems [3] relate the solutions of two equations only. Another difference is the fact that the initial state $u_{0}$ of the maximal solution $r\left(s, t_{0}, u_{0}\right)$ of (2.3) depends upon the parameter $t$. Because of these reasons, we call this result the "variational comparison theorem". The structure of the variational comparison theorem may play a very important role in studying the qualitative behavior of perturbed system (2.2). In some situations, it might be more useful than the usual variation of constants formula as well as comparison theorems [3]. In the succeeding section, we shall justify our remark by exhibiting examples.

3. Applications and examples. In this section, we shall give a few applications of our basic result developed in $\$ 2$.

To begin with, let us show, as a simple application, that a result on stability analogous to Theorem 4.4.6 in [3] may be formulated from the variational comparison theorem.

Because of the nature of the variational comparison theorem, we need to introduce a new notion of stability with respect to auxiliary equation (2.3) and unperturbed equation (2.1).

Definition 3.1. The trivial solution of (2.1) is said to be $V$-equistable with respect to (2.3) if, for each $\epsilon>0, t_{0} \in R^{+}$, there exists a positive 
function $\delta=\delta\left(t_{0}, \epsilon\right)$ that is continuous in $t_{0}$ for each $\epsilon$ such that the inequality $\left\|x_{0}\right\| \leq \delta$ implies $\sum_{i=1}^{m}\left|r_{i}\left(t, t_{0}, u_{0}\right)\right|<\epsilon$, for $t \geq t_{0}$, where $u_{0}=$ $V\left(t, x\left(t, t_{0}, x_{0}\right)\right)$, where $V \in C\left[R^{+} \times R^{n}, R^{m}\right]$, and where $x\left(t, t_{0}, x_{0}\right)$ and $r\left(t, t_{0}, u_{0}\right)$ are solutions of (2.1) and (2.3) respectively.

Other definitions of stability can be formulated similarly.

Theorem 3.1. Let all hypotheses of Theorem 2.1 be satisfied. Further assume that $f, R, g$ and $V$ satisfy the hypotheses:

$\left(\mathrm{H}_{7}\right) f(t, 0) \equiv 0 \equiv R(t, 0)$,

$\left(\mathrm{H}_{8}\right)$ for $(t, x) \in R^{+} \times R^{n}, c(\|x\|) \leq \Sigma_{i=1}^{m} V_{i}(t, x) \leq d(\|x\|)$, where $c, d \epsilon$ $C\left[R^{+}, R^{+}\right], c(0)=d(0)=0$ and $c(r)$ and $d(r)$ are increasing in $r$.

Then, the V-equistability or V-asymptotic stability of the trivial solution of (2.1) implies the stability or asymptotic stability of the trivial solution of (2.2).

Proof. The proof may be constructed on the basis of the proof of Theorem 2.1 and following the argument in Theorem 4.4.6 in [3]. We therefore leave the details.

In the following, we shall give two examples to justify Remark 2.4.

Example 3.1. Consider the scalar differential equation

$$
x^{\prime}=-1 / 2 x^{3}, \quad x\left(t_{0}\right)=y_{0},
$$

and its corresponding perturbed equation

$$
y^{\prime}=-1 / 2 y^{3}+R(t, y), \quad y\left(t_{0}\right)=y_{0},
$$

where $R$ satisfies $\left(\mathrm{H}_{2}\right), R(t, 0) \equiv 0$ and the relation

$$
y R(t, y) \leq \lambda(t)|y|^{2} \quad \text { for }(t, y) \in R^{+} \times R,
$$

where

$$
\lambda \in C\left[R^{+}, R^{+}\right] \cap L_{1}[0, \infty) .
$$

Thus $x\left(t, t_{0}, x_{0}\right)=y_{0} /\left[1+\left(t-t_{0}\right) y_{0}^{2}\right]^{1 / 2}$ and $\phi\left(t, t_{0}, y_{0}\right)=$ $1 /\left[1+\left(t-t_{0}\right) y_{0}^{2}\right]^{3 / 2}$. It is easy to see that the trivial solution of (3.1) is uniformly asymptotically stable. Then taking $V(t, x)=1 / 2|x|^{2}$, we see that

$$
\frac{d V}{d s}(t, x(t, s, y(s))) \leq \lambda(s) V(t, x(t, s, y(s))), \quad t \geq s \geq t_{0},
$$

where $x(t, s, y(s))$ is the solution of (3.1) through $(s, y(s))$ and $y\left(s, t_{0}, y_{0}\right)$ $=y(s)$ is any solution of (3.2). The auxiliary equation is $u^{\prime}=\lambda(t) u$. Thus $r\left(t, t_{0}, u_{0}\right)=u_{0} \exp \left[\int_{t_{0}}^{t} \lambda(s) d s\right]$, where $u_{0}=1 / 2\left|x\left(t, t_{0}, y_{0}\right)\right|^{2}$. From (3.4) and the uniform asymptotic stability of $x \equiv 0$ of (3.1) we have that $x \equiv 0$ of (3.1) is $V$-uniformly a symptotically stable. Hence by Theorem 3.1 , it follows that the trivial solution of (3.2) is uniformly asymptotically stable. 
Remark 3.1. Example 3.1 shows that the uniform asymptotic stability of $x \equiv 0$ of (2.1) plus the uniform stability of $u \equiv 0$ of (2.3) implies $V$-uniform asymptotic stability of $x \equiv 0$ of (2.1).

Remark 3.2. It is known that if $x \equiv 0$ of (2.1) is uniformly asymptotically stable then we can find a Lyapunov function which can be used to study (2.2). For more details see [3]. However, use of the Lyapunov function forces us to take the norm of the perturbed term $R(t, y)$. This fact makes it impossible to deduce any information about the asymptotic stability of (2.2), even though it is uniformly asymptotically stable. One can easily justify this fact from our previous example. This shows a gain over usual comparison theorems. It is also well known [2] that if $\sigma<0$ and $R(t, y)=$ $o(y)$, then $y \equiv 0$ of (2.2) is uniformly asymptotically stable. This result has been proved in [2] by using an analogue of the variation of constants formula for nonlinear systems. By this method, it is not possible to prove the following statement: "If $\sigma=0, R(t, 0)=o(y)$ and $x \equiv 0$ of (2.1) is uniformly asymptotically stable, then $y \equiv 0$ of (2.2) is uniformly asymptotically stable." Details may be found in [2], [3]. Note that Example 3.1 satisfies all the hypotheses of the above statement. This indicates an advantage of our "variational comparison theorem" over usual variation of constants formula.

Example 3.2. Consider the differential equation

$$
x^{\prime}=x /(1+t), \quad x\left(t_{0}\right)=y_{0},
$$

and its corresponding perturbed equation

$$
y^{\prime}=y /(1+t)+R(t, y), \quad y\left(t_{0}\right)=y_{0},
$$

where $R(t, y)$ satisfies $\left(\mathrm{H}_{2}\right), R(t, 0) \equiv 0$ and the relation

$$
y R(t, y) \leq-\alpha|y|^{2} \text { for }(t, y) \in R^{+} \times R
$$

and any $a>0$. Thus $x\left(t, t_{0}, y_{0}\right)=y_{0}(1+t) /\left(1+t_{0}\right), \phi\left(t, t_{0}, y_{0}\right)=$ $(1+t) /\left(1+t_{0}\right)$. It is easy to observe that $x \equiv 0$ of (3.6) is unstable. We take $V(t, x)=1 / 2|x|^{2}$. After computations, we get

$$
\frac{d V}{d s}(t, x(t, s, y(s))) \leq-\alpha V(t, x(t, s, y(s))), \quad t \geq s \geq t_{0},
$$

where $x(t, s, y(s))$ and $y\left(s, t_{0}, y_{0}\right)$ are solutions of (3.6) and (3.7) respectively. The auxiliary equation is $u^{\prime}=\alpha u$. Thus $r\left(t, t_{0}, u_{0}\right)=u_{0} e^{-\alpha\left(t-t_{0}\right)}$ with $u_{0}=1 / 2\left|x\left(t, t_{0}, y_{0}\right)\right|^{2}$. From (3.8), it follows that $x \equiv 0$ of (3.6) is $V$ uniformly asymptotically stable. Hence by Theorem 3.1 , it follows that $y \equiv 0$ of (3.7) is uniformly asymptotically stable.

Remark 3.3. Example 3.2 shows that $x \equiv 0$ of (2.1) may be $V$-uniformly asymptotically stable, even though $x \equiv 0$ of (2.1) is unstable. This is 
another advantage of the variational comparison theorem over both methods. In our earlier work [1], we need at least stability of $x \equiv 0$ of (2.1) to study the asymptotic stability of $y \equiv 0$ of (2.2). In the light of our example, we do not need that too. This is certainly a great advantage for studying constantly acting perturbations.

Remarks 3.1-3.3 exhibit that $y \equiv 0$ of (2.2) may be stable or asymptotically stable whether $x=0$ of $(2.1)$ is stable or not. This opens a new avenue for studying differential equations $x^{\prime}=F(t, x)$ for which one may not be able to study its qualitative behavior by Lyapunov or variational methods. However, if one can write $F(t, x)=f(t, x)+R(t, x)$, where $f$ and $R$ satisfy $\left(\mathrm{H}_{1}\right)$ and $\left(\mathrm{H}_{2}\right)$ respectively, then the variational comparison theorem gives-a hope for studying the behavior of $x^{\prime}=F(t, x)$.

In none of the applications of "variational comparison theorem" have we used the full force of the theorem. We have also not given any applications of the generalized variation of constants formula which will have more scope for studying stochastic perturbations. By viewing the scope of these theorems, we should also be able to obtain results on asymptotic equivalence, conditional stability, partial stability and boundedness.

\section{REFERENCES}

1. S. Bernfeld, G. Ladde and V. Lakshmikantham, On the classes of differential systems with the desired behavior, Rend. Circ. Mat. Palermo (2) 21 (1972), 85-97. MR 48 \#2505.

2. F. Brauer, Perturbations of nonlinear systems of differential equations. I, J. Math. Anal. Appl. 14 (1966), 198-206. MR 33 \#359.

3. V. Lakshmikantham and S. Leela, Differential and integral inequalities, Theory and Applications, Vols. I, II, Academic Press, New York, 1969.

DEPARTMENT OF MATHEMATICS, ST ATE UNIVERSITY OF NEW YORK AT POTSDAM, POTSDAM, NEW YORK 13676 